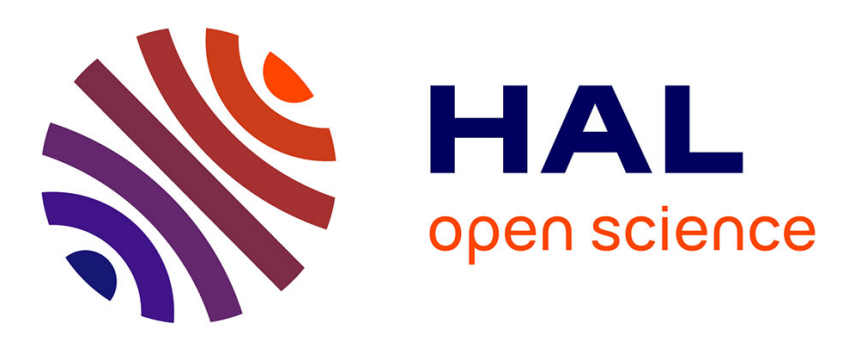

\title{
Automatic Nacre Thickness Measurement of Tahitian Pearls
}

\author{
Alban Gabillon, Martin Loesdau, Sébastien Chabrier
}

\section{To cite this version:}

Alban Gabillon, Martin Loesdau, Sébastien Chabrier. Automatic Nacre Thickness Measurement of Tahitian Pearls. Lecture Notes in Computer Science, 9164, Springer Verlag, pp.446-455, 2015, 10.1007/978-3-319-20801-5_49. hal-03132306

\section{HAL Id: hal-03132306 https://hal-upf.archives-ouvertes.fr/hal-03132306}

Submitted on 5 Feb 2021

HAL is a multi-disciplinary open access archive for the deposit and dissemination of scientific research documents, whether they are published or not. The documents may come from teaching and research institutions in France or abroad, or from public or private research centers.
L'archive ouverte pluridisciplinaire HAL, est destinée au dépôt et à la diffusion de documents scientifiques de niveau recherche, publiés ou non, émanant des établissements d'enseignement et de recherche français ou étrangers, des laboratoires publics ou privés. 


\title{
Automatic Nacre Thickness Measurement of Tahitian Pearls
}

\author{
Martin Loesdau, Sébastien Chabrier, Alban Gabillon \\ Laboratoire d'Excellence CORAIL \\ Géopôle du Pacifique Sud EA4238 \\ Université de la Polynésie française, Tahiti \\ \{martin.loesdau, sebastien.chabrier, alban.gabillon\}@upf.pf
}

\begin{abstract}
In this paper a methodology for an automatized measurement of the nacre thickness of Tahitian pearls is presented. An adapted snake approach as well as our own developed circle detection algorithm are implemented to extract the nacre boundaries out of X-ray images. The results are validated by experts currently performing manually the obligatory nacre thickness control for millions of Tahitian pearls that are exported each year. Equivalent articles propose methods suitable for round pearls, whereas this paper contains methods to evaluate the nacre profile of pearls independently of their shape. As the algorithms are not specifically parametrized for Tahitian pearls, the methods can be adapted for quality assessment of other pearls as well.
\end{abstract}

Keywords: Pearl Classification, X-ray Image Analysis, Active Contours, Circle Detection

\section{Introduction}

The Tahitian pearl is a precious natural gem that is cultivated in the clear warm lagoons of French Polynesia. On the international market the pearl is known under the name 'Queen of Pearls', due to its high quality and the large diversity of different color nuances. To keep its high reputation, the French Polynesian government introduced an obligatory quality control for each pearl that is supposed to be exported. This obligatory control is conducted by the administration of marine and mining resources (Direction des Ressources Marines et Minières, DRMM), where the quality of a pearl is evaluated by its form, size, color, luster, surface quality and nacre thickness. The evaluation is done manually by experts, which is a time consuming process, especially seeing the large amount of pearls that are exported each year (over 11 million in 2014, tendency increasing). As the pearl is the first source of export income in French Polynesia (export volume of over 70 million Euro in 2014), one goal of our project is to support this important branch by implementing a computer vision based quality control. An automatized quality assessment can help to guarantee a fast and stable export procedure, which is in the interest of the local pearl farmers as well as the French Polynesian government.

adfa, p. 1, 2011.

(C) Springer-Verlag Berlin Heidelberg 2011 

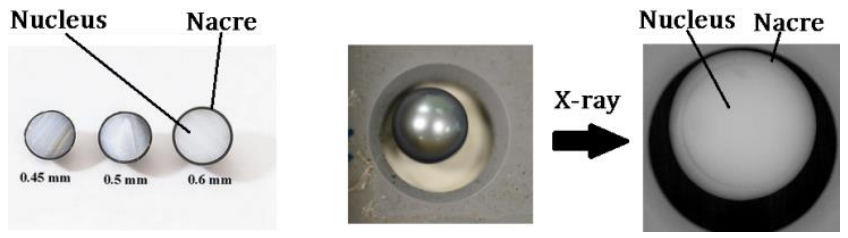

Fig. 1. A cut through three Tahitian pearls (left), a Tahitian pearl positioned in a borehole of a wooden plate for X-raying (middle) and the resulting X-ray image (right).

The Tahitian pearl is cultivated by inserting an artificially formed sphere, the nucleus, into a Black-Lip Pearl Oyster (Pinctada margaritifera). The pearl grows afterwards as a result of a biological defense mechanism of the oyster that 'neutralizes' this foreign substance by building layers of nacre around it (see Fig. 1 on the left for a cut through 3 Tahitian pearls). One of the quality parameters to control is the minimal nacre thickness, a parameter that has to be evaluated by imaging the internal structure of the pearl. For this purpose the DRMM uses X-ray machines. The pearls are stored in boreholes of a wooden plate that is placed in the machine for image capturing (Fig. 1 in the middle). Afterwards an employee evaluates manually the numeric image taken separately for each pearl (Fig. 1 on the right). An obligatory export criterion for a Tahitian pearl is that its minimal nacre thickness has to exceed $0.8 \mathrm{~mm}$. The manual evaluation of the X-ray images serves accordingly the purpose of rejecting pearls with a minimal nacre thickness lower than $0.8 \mathrm{~mm}$ from exportation.

In this paper our methodology to automatize the measure of the nacre profile out of $\mathrm{X}$-ray images is presented. The crucial regions to detect to automatize this measurement are visualized in Fig. 2. The example in the left column shows a round pearl situated completely in the plates borehole (green circle). The outer boundary of the pearl (blue line) and the nucleus (red circle) have to be detected and the distance between both describes the nacre thickness profile. The second column shows a pearl with a cavity inside. As the cavity must not contribute to the thickness measurement, the inner boundary of the nacre has to be extracted additionally (cyan line). The distance between the inner and the outer boundary describes the nacre thickness. The third example shows a pearl, whose outer boundary surpasses completely the borehole, leading to superposed gradients of the borehole and the inner structure of the pearl, a complication that will be discussed in section four.
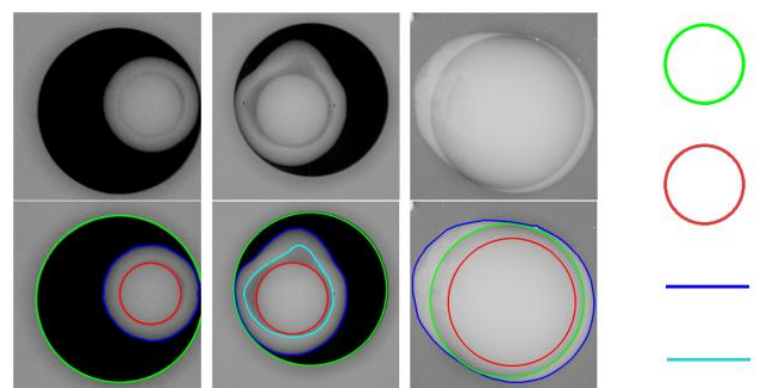

Borehole

Nucleus

Outer boundary

Inner boundary

Fig. 2. Three example images (first row) and the crucial regions to detect (second row). 
In the next section, related work concerning pearl quality assessment based on nacre thickness, color and shape is described. Section three contains our methods to measure the nacre thickness of Tahitian pearls. In three subsections the stages of extracting the inner and outer boundary of the nacre with active contours, as well as extracting the nucleus with circle detection are described. The fourth subsection contains the final measurement of the nacre profile out of the previously extracted information. In section four perspectives concerning the improvement and further validation of our methods as well as suggestions to improve the image configuration can be found, followed by the conclusion in section five.

\section{Related Work}

Even though the international pearl market yields large profit in several countries, scientific work in the domain of computer science to improve this industry is rare. An article proposing a method for an automatized nacre thickness measurement can be found under [1]. Optical coherence tomography (OCT), a shallow-depths laser imaging technique, is used to generate greylevel images of the internal structure of a pearl. The OCT images undergo denoising, edge detection and median filtering, to detect the edges of the outer boundary of the nacre. According to the assumption that the outer boundary of the nacre is round, the final boundary is obtained by fitting a circle to the obtained edges using the least-square method. The edge pixels for the inner boundary are identified with Support Vector Machine and the results are treated the same way as for the outer boundary. Further developments of this method are described in $[2,3]$. The approaches are based on the assumption that the nacres' boundaries are round, which covers only one of several classes concerning the Tahitian pearl. The left image in Fig. 3, taken from [1], shows two circles in blue that approximate the inner and the outer boundary of a pearl, as a result of the described algorithm. In the middle an image of Tahitian pearls that are controlled for export at the DRMM can be seen, most of them with a 'baroque' shape that cannot be approximated by circles. On the right a typical X-ray image of a pearl with a 'baroque' shape is shown to further illustrate the need for another approach to automatically measure the nacre thickness of Tahitian pearls independently of its shape.
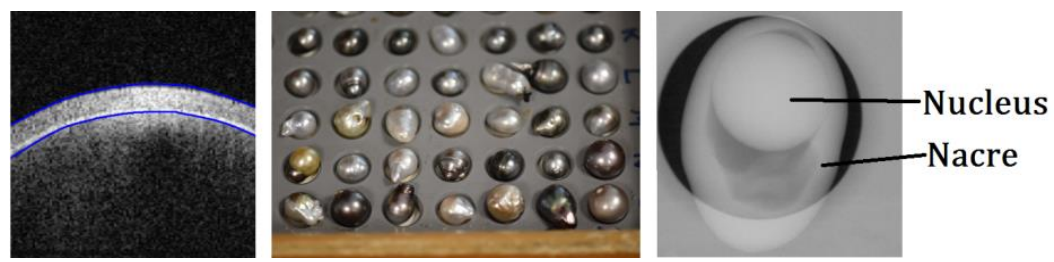

Fig. 3. One result image of the nacre thickness measurement from [1] (left), several Tahitian pearls with 'a baroque' shape prepared for X-raying (middle) and an X-ray image of a typical Tahitian 'baroque' pearl (right). 
Further related articles concern the quality assessment of pearls based on color and shape. In [4-7] the relation between the physical properties of a pearl and its human evaluation are investigated. A model is built which aims to support automated inspection systems in regards of for example the spectrum, diffusion and position of a light source. In [8] the pearls shape is described with Zernike moments and afterwards classified with a fuzzy membership function, while an approach to classify the pearls color is presented in [9].

\section{Measuring the nacre thickness}

To measure the nacre thickness out of X-ray images the outer and the inner boundary of the nacre have to be extracted. Our methodology consists of three stages: (i) detecting the outer boundary of the nacre, (ii) detecting the nucleus, and (iii) detecting the inner boundary of the nacre. The methods for these stages will be presented in the following three sections. In the fourth section the final measurement of the nacre thickness out of the obtained information is described.

\subsection{Detecting the Outer Boundary}

As the intensity and the gradient of the outer boundary of the pearls in X-ray images vary largely, classical edge detection (such as Canny, Sobel or Prewitt) for the detection of the outer boundary failed in first tests. A more efficient approach is to use the active contour approach 'snakes' [10]. This advanced edge integrator consists of a curve that is moved by image-dependent external forces while its form keeps a certain degree of 'smoothness'. The mathematical idea is to maintain equilibrium between internal and external energy defined over the whole curve at each iteration. The according formula is described as:

$$
\int\left(\alpha(s)\left(\frac{d(x(s))}{d s}\right)^{2}+\beta(s)\left(\frac{d^{2}(x(s))}{d s^{2}}\right)^{2}+F(s)\right) d s=0 .
$$

The first two terms contain the first two derivatives of the curve that describe the smoothness of its contour (internal energy). The third term is the force that moves the curve (external energy), usually a gradient calculated over the whole image. This integral describes an optimization problem that can be solved with the Euler-Lagrange formalism. A simple numeric implementation of the solution can be described by

$$
\vec{X}_{t+1}=(\overline{\bar{I}}-\overline{\bar{A}})^{-1}\left(\vec{F}+\vec{X}_{t}\right) .
$$

In this formula $\overline{\bar{I}}$ is the identity matrix, $\overline{\bar{A}}$ is a matrix that contains the weighted second and fourth derivative of the curve at time $t, \vec{F}$ is a force vector that moves the curve and $\vec{X}$ is a vector that contains the $\mathrm{x}$-coordinates of the curve at time $t$, respectively, as the algorithm is iterative at time $t+1$. The algorithm (executed for the $\mathrm{x}-$ and $\mathrm{y}-$ coordinates) results in a moving curve that stops moving when the external and internal energy at each point are in equilibrium. 

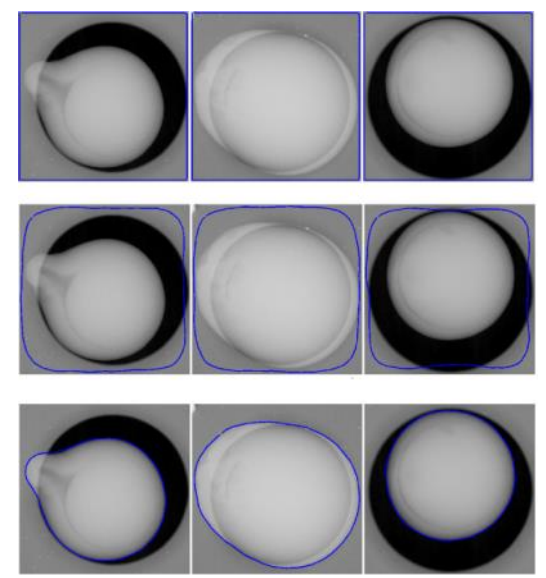

Fig. 4. The initial snake contour for the outer boundary detection in blue (top), after several iterations (middle) and the final result (bottom).

The implementation for detecting the outer boundary of the nacre is the following: the initial contour is set to the outer boundary of the image, assuring that the pearl is situated inside the contour (Fig. 4 first row in blue color for three example images). The normal vectors at each point of the curve point to the inside of the curve. The moving force is a negative 'balloon' force, meaning the curve is constantly shrinking in direction of its normal vectors (for a description of the balloon force please see [11]). This force is diminished if the curve touches positive image gradients (dark pixel to light pixel) in direction of its normal vectors. The effect of this configuration can be seen in Fig. 4 second and third row: while the curve in each image passes the strong gradient between the light and the dark background (negative gradient) it stops at the positive gradient that describes the outer boundary of the pearl. These three examples contain different possibilities of pearl position. While the pearl on the right is situated completely in the borehole of the plate on which the pearls are positioned (see as well Fig. 1), the image on the left shows a pearl whose boundary surpasses partially the hole. The image in the middle is an example of a pearl whose boundary surpasses completely the hole. Even though the local gradients of the pearl boundaries are different, no case differentiation has to be done, as for all three configurations the same algorithm with the same parametrization was used.

After obtaining the outer boundary of the pearl, the second stage consists of detecting the nucleus within the pearl.

\subsection{Detecting the Nucleus}

As described in the introduction, the nucleus is an artificially formed sphere, which appears as a circle in X-ray images. The goal of this stage is accordingly classical circle detection. One of the most popular approaches is the circular Hough transform [12]. Even though with high performance, this 'brute force' approach is time and memory consuming. As center and radius of the nucleus are a priori unknown, each 
pixel within the outer boundary has to be considered as potential center pixel for all radii in this approach. The low gradients and the nacre thickness dependent intensity makes pixel preselection with edge detection difficult. Using the snake approach instead does not guarantee that the result will be a circle, especially if the boundary of the nucleus is only partially visible. For the purpose of the nucleus detection we developed a heuristic approach that consists of an artificial circle with a variable radius that is moved by image gradient forces. The basic idea is: if an artificial circle stays all time in a circular object to detect, while its radius is increased, it will at one time fully cover the outer boundary of the circular object. For this application it means that an initial artificial circle has to move into the nucleus and stay in it while its radius is constantly increased. At a certain moment the artificial circle will fully cover the outer boundary of the nucleus. The mathematical formula satisfying this movement can be denoted as

$$
\vec{F}=\sum_{i=1}^{m} f_{i} \vec{n}_{i}
$$

In this formula $\vec{n}_{i}$ denotes the inside pointing normal vector of point $i$ of the artificial circle and $f_{i}$ the local gradient force at this point (positive from dark to light pixels in direction of the normal vector). The sum over all $m$ points of the circle determines its moving direction $\vec{F}$. Each time the center of the moving circle touches the same pixel for the second time its radius is increased. The implementation for one of the example images is visualized in Fig. 5. On the top left the initial circle is shown with its normal vectors at each point weighted by the local gradient (blue arrows). Due to the spherical form of the nucleus, the gradients at the border are stronger, resulting according to Eq. 3 in a moving direction (black arrow) pointing to the center of the nucleus. As the border between the nucleus and the nacre is barely visible, a red arrow points for clarification at the partial border. The bigger circle with strong gradients in this image belongs to the borehole of the plate where the pearl is placed for X-raying (see as well Fig. 1 and Fig. 2). At the bottom of Fig. 5 is the average of all local gradients of all circle points over each movement visualized in blue. Each time the radius is increased is marked by a vertical black line.

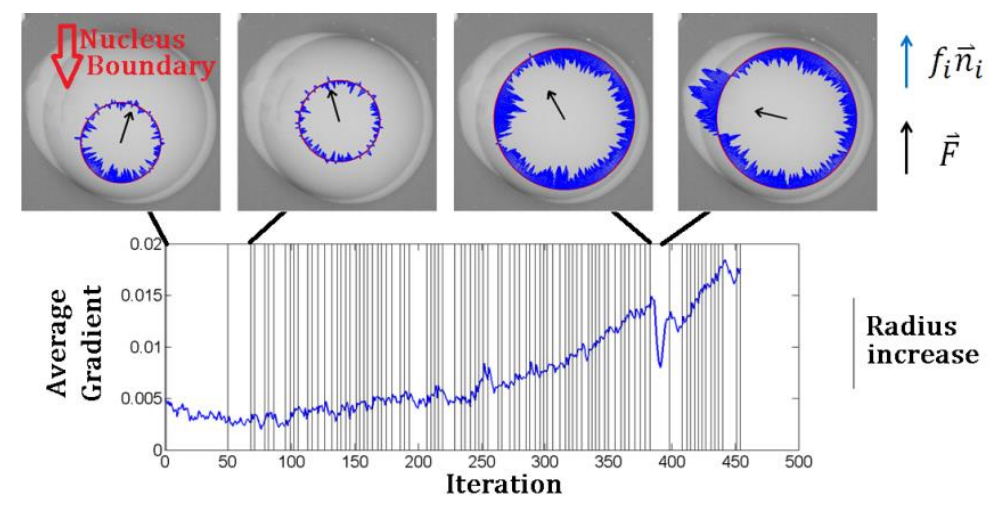

Fig. 5. Four different iteration stages of our circle detection algorithm (top) and the average gradient of each circle point at each iteration (bottom). 

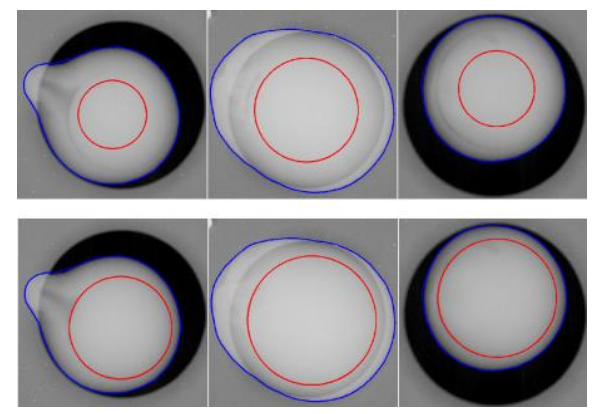

Fig. 6. The initial artificial circle of the implemented algorithm for the nucleus detection in red (top) and the detected nucleus (bottom).

After the first two radius increases, the circle moved further inside the nucleus close to its center (second image on the top). The third image shows the circle and its weighted normal vectors at the moment it fully covers the boundary of the nucleus. Almost all gradients are positive and at a local maximum. A few movements later the circle surpasses this boundary, resulting in an abrupt change of gradient directions in this area (fourth image), due to the beginning of the nacre area. This moment, even though the boundary of the nucleus is only partially visible, can be clearly detected in the trend of the average gradients (on the bottom of Fig. 5). The automatically detected nuclei of all three example pearls with this algorithm are shown in Fig. 6. On the top are the circles initialized at the geometric center of the previously detected outer boundary and the results are on the bottom. For the two example pearls in the right two columns, all necessary information to calculate the nacre thickness is obtained (the inner nacre boundary is equivalent to the boundary of the nucleus). The pearl on the left however, has a cavity inside the nacre which must not contribute to the nacre thickness measurement. Therefor a third stage of detecting the inner boundary has to be executed.

\subsection{Detecting the Inner Boundary}

In Fig. 7 on the left the result of the first two stages for one of the example images of the previous sections is shown. Within the nacre a slightly darker region can be identified. This region is a cavity within the pearl. Accordingly this region must not contribute to the nacre thickness measurement. For the same reasons as before, the active contour approach 'snakes' can as well be used for this stage.
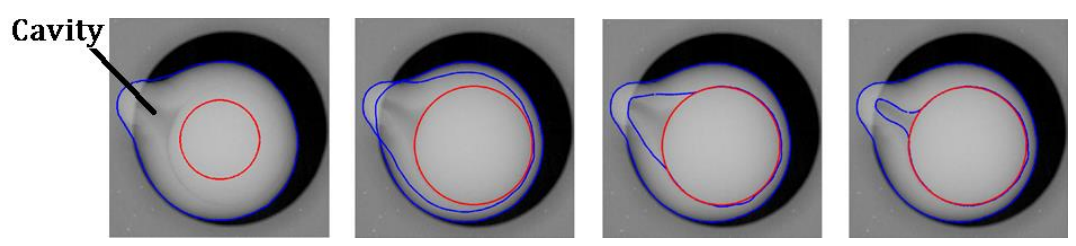

Fig. 7. Different stages for the detection of the inner boundary of the nacre in case of a cavity within the pearl. The final result is on the right. 
In this implementation the initial contour is set identical to the detected outer boundary of the pearl. The moving force is like previously a negative 'balloon' force, resulting in a constant shrinking of the curve. This time the shrinking is diminished if the curve touches negative gradients or the detected nucleus. The right three images in Fig. 7 visualize the execution of the algorithm. The final result approximates the inner boundary of the nacre. For the other two pearls seen in the previous section, the inner boundary is equivalent to the detected boundary of the nucleus, as their inner structure possesses no cavities. As by now all necessary information for the three pearls are extracted, the actual nacre thickness can be evaluated.

\subsection{Measuring the thickness}

To evaluate the nacre thickness the distance between the outer and inner boundary is calculated at every point, which describes the nacre thickness profile of the whole pearl (Fig. 8 in the middle). The last step is to identify the regions of the nacre profile that are thinner than the minimal authorized nacre thickness of $0.8 \mathrm{~mm}$ for Tahitian pearls to be exported. The areas of the nacre greater than this margin are colored in green while the thinner areas are colored in red (Fig. 8 on the bottom). The results were validated by employees of the DRMM.
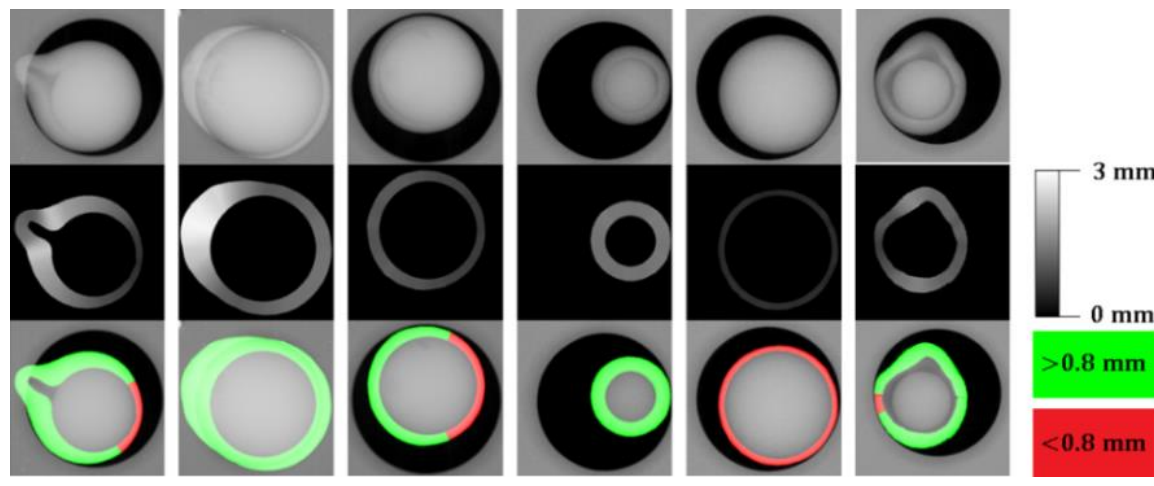

Fig. 8. Original images (top), automatically calculated nacre profiles (middle) and a visualization of areas lower than the minimal allowed nacre thickness for exporting a Tahitian pearl (bottom, areas lower than $0.8 \mathrm{~mm}$ in red).

\section{$4 \quad$ Perspectives}

As by this case study the used methods are generally validated as suitable, the next step is to acquire a large amount of test images to further improve and adapt the used algorithms. We are currently in contact with the DRMM to obtain a set of several hundred X-ray images together with manual evaluation results. Furthermore several special cases exist as shown in Fig. 9. The image on the left shows a pearl with two inner boundaries, while the image in the middle shows a pearl whose inner structure does not allow a visual discrimination between nucleus and nacre. 

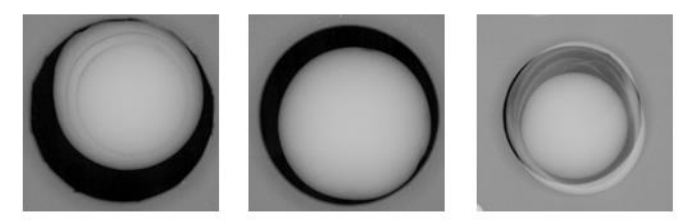

Fig. 9. Special cases of inner nacre structure: two inner boundaries (left), inner boundary not visible (middle) and complex inner structure (right).

The image on the right shows a pearl whose inner structure is highly complex. The goal is to automatically identify the special cases to at least, if an automatic detection is impossible, avoid false detections.

Another perspective concerns the plate that is used to position the pearls for X-raying. Currently the pearls are placed in boreholes of a wooden plate (see Fig. 2 and Fig. 3). These additional gradients superposed on the internal structure of the nacre complicate and decelerate the automatic analysis, as well as the manual evaluation by experts. We proposed two possibilities to the DRMM how to avoid these constellations. One possibility is to enlarge the boreholes of the plate so that every pearl is always situated completely within the borehole (Fig. 10 in the middle). Another possibility is to change the profile of the plate completely, so that the intensity of the exiting X-rays is at every point equal (Fig. 10 on the right).

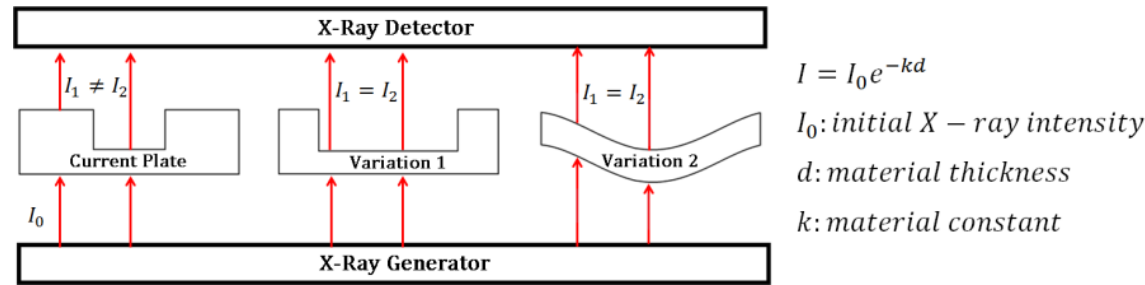

Fig. 10. Profile schema of the currently used wooden plate (left) and variations with enlarged boreholes (middle) and a complete profile change (right) to avoid superposed gradients.

\section{Conclusion}

In this paper we presented a methodology to automatically measure the nacre thickness of Tahitian pearls out of X-ray images. This work aims to support and improve the French Polynesian pearl business by automatizing an obligatory measurement that is currently done manually. Equivalent articles cover only one particular case of round pearls, whereas this paper contains a more general approach for pearls of different shape. An adapted snake approach as well as our own developed circle detection algorithm are used to extract the necessary information from the X-ray images. Our results were validated by employees of the administration of marine and mining resources of French Polynesia, a governmental institution that is in charge of the obligatory nacre thickness control of Tahitian pearls that are exported. The automatic detection is performed in real-time, a necessary requirement for the quality assessment. 


\section{Acknowledgements}

We thank Cedrik Lo and Vaihere Mooria from the DRMM for providing us access to their facilities and for the supply of manually classified X-ray images. We thank as well all involved employees of the DRMM for their help and for sharing their knowledge and professional experience to support our work.

\section{References}

1. Lei, Ming, et al. "Automated thickness measurements of pearl from optical coherence tomography images." Hybrid Intelligent Systems, 2009. HIS'09. Ninth International Conference on. Vol. 1. IEEE, 2009.

2. Sun, Yankui, and Ming Lei. "Automated thickness measurements of nacre from optical coherence tomography using polar transform and probability density projection." Intelligent Signal Processing and Communication Systems (ISPACS), 2010 International Symposium on. IEEE, 2010.

3. Liu, Jing, Xiao Lin Tian, and Yan Kui Sun. "Pearl Thickness Measurements from Optical Coherence Tomography Images." Applied Mechanics and Materials 421 (2013): 415-420.

4. Dobashi, Toshimasa, et al. "Implementation of a pearl visual simulator based on blurring and interference." Knowledge-Based Intelligent Electronic Systems, 1998. Proceedings KES'98. 1998 Second International Conference on. Vol. 3. IEEE, 1998.

5. Nagata, Noriko, et al. "Modeling and visualization for a pearl-quality evaluation simulator." Visualization and Computer Graphics, IEEE Transactions on 3.4 (1997): 307-315.

6. Nagata, Noriko, Mitsuhito Kamei, and Teruo Usami. "Transferring Human Sensibilities to Machines-Sensitivity Analysis of Layered Neural Networks and Its Application to Pearl Color Evaluation." MVA. 1994.

7. Nagata, Noriko, et al. "Image analysis and synthesis using physics-based-modeling for pearl quality evaluation system." Image Analysis and Processing. Springer Berlin Heidelberg, 1997.

8. Li, Bin, et al. "A classification method of pearl shape based on Zernike moment." Wavelet Analysis and Pattern Recognition, 2007. ICWAPR'07. International Conference on. Vol. 3. IEEE, 2007.

9. Tian, Chunyu. "A computer vision-based classification method for pearl quality assessment." Computer Technology and Development, 2009. ICCTD'09. International Conference on. Vol. 2. IEEE, 2009.

10. Kass, Michael, Andrew Witkin, and Demetri Terzopoulos. "Snakes: Active contour models." International journal of computer vision 1.4 (1988): 321-331.

11. Cohen, Laurent D. "On active contour models and balloons." CVGIP: Image understanding 53.2 (1991): 211-218.

12. Duda, Richard O., and Peter E. Hart. "Use of the Hough transformation to detect lines and curves in pictures." Communications of the ACM 15.1 (1972): 11-15. 